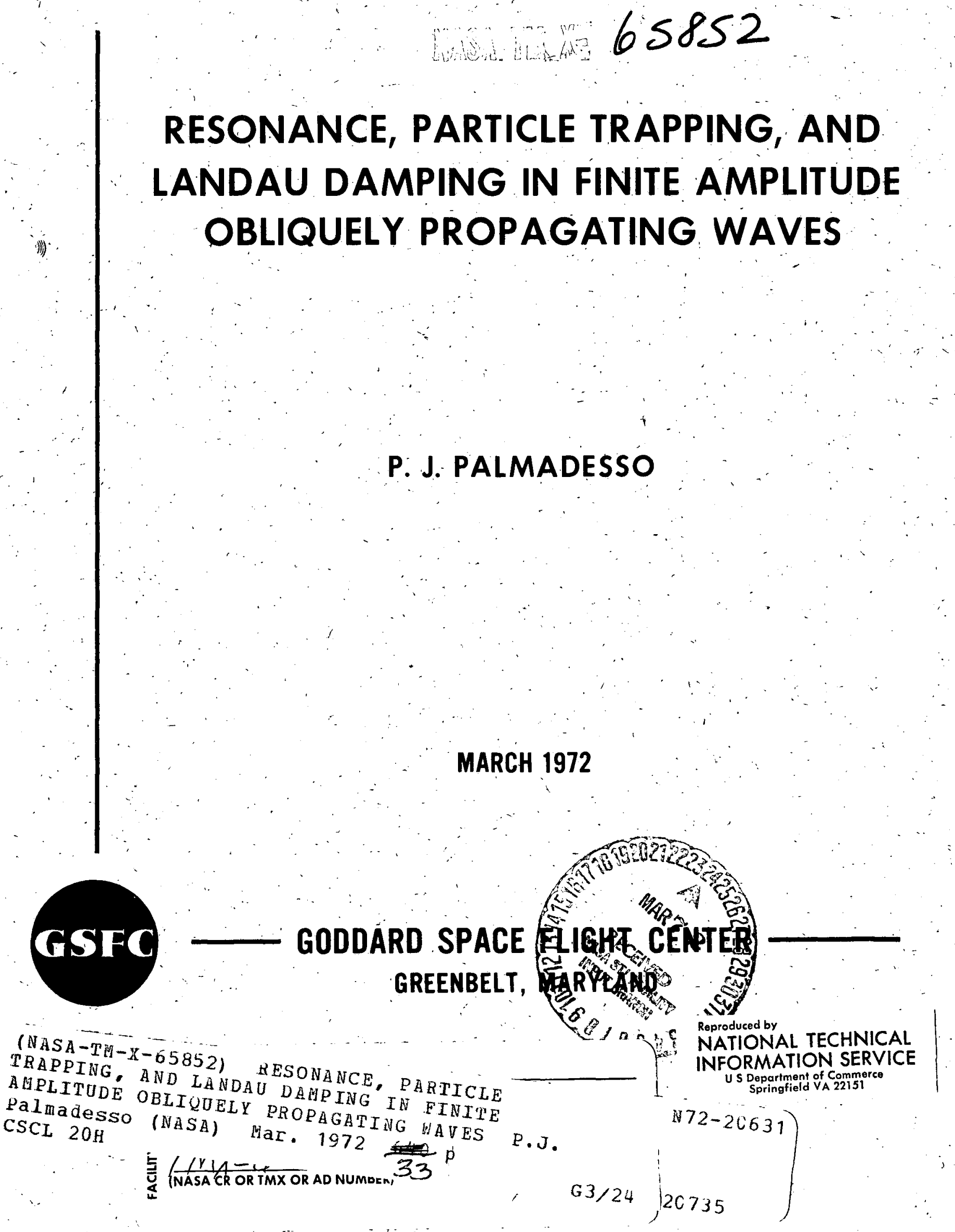


RESONANCE, PARTICLE TRAPPING, AND LANDAU DAMPING IN FINITE AMPLITUDE OBLIQUELY PROPAGATING WAVES

\author{
P. J. Palmadesso \\ Goddard Space Flight Center \\ National Aeronautics and Space Administration \\ Greenbelt, Maryland 20771
}

March 1972

GODDARD SPACE FLIGHT CENTER

Greenbelt, Maryland 


\title{
RESONANCE, PARTICLE TRAPPING, AND LANDAU DAMPING IN FINITE AMPLITUDE OBLIQUELY PROPAGATING WAVES
}

\author{
P. J. Palmadesso \\ Goddard Space Flight Center \\ Greenbelt, Maryland
}

\begin{abstract}
The equations of motion for a particle in resonance with a small finite amplitude wave $(\omega, \mathrm{k})$ are solved approximately, using secularity free perturbation theory. The wave propagates at an arbitrary angle to a uniform background magnetic field, $\underset{\sim}{\mathbf{B}}$, in an infinite collisionless plasma. The wave fields include a longitudinal electrostatic component and elliptically polarized transverse ; electric and magnetic components. The trajectories of trapped and resonant untrapped particles are described, for each of the possible wave-particle resonances defined by the condition $\mathrm{k}_{\mathrm{z}} \mathrm{V}_{\mathrm{z}}-\omega \approx \mathrm{Nq}_{0} / \mathrm{m}$, where $\mathrm{N}$ is an integer. These trajectories are used to construct an estimate of the nonlinear time dependent Landau damping rate of the wave.
\end{abstract}




\section{INTRODUCTION}

The trapping of particles by finite amplitude narrowband waves leads to a number of important nonlinear effects. Particle trapping is responsible for the saturation of the Landau damping/amplification mechanism in the nonlinear regime. ${ }^{1,2}$ Electrostatic trapped particle instabilities, ${ }^{3-6}$ and the triggering of V.L.F. emissions by finite amplitude whistlers in the magnetosphere ${ }^{7,8}$ provide other examples of effects in which trapped particles play a major role.

Explanations of these phenomena depend heavily on a knowledge of the trajectories of individual resonant particles, i.e., trapped and nearly trapped particles, in the fields of the primary wave. In all of the examples mentioned above, the primary wave is either an electrostatic wave or a circularly polarized transverse wave propagating parallel to a background magnetic field. The trajectories of particles in fields of these types are well known. ${ }^{1,9}$

In this paper we obtain relatively simple approximate analytic expressions which describe the motion of single particles in resonance with a wave of somewhat more general type. We consider a small finite amplitude wave propagating at an arbitrary angle to a uniform background magnetic field in an infinite collisionless plasma. The wave has both longitudinal electrostatic and transverse electromagnetic components.

An immediate application of the results of the trajectory analysis is possible. The approximate orbit expressions for resonant motion in an oblique wave have a 
form which is similar in some important respects to the corresponding expressions for motion in an electrostatic wave or a parallel propagating transverse wave. By recognizing this, one is able to obtain in a simple way an estimate of the time dependent collisionless damping coefficient for a finite amplitude oblique wave. One finds that after some initial exponential damping the wave executes damped amplitude fluctuations. In the absence of instabilities, the wave amplitude would ultimately become constant.

We begin in Sec. II by solving the equations of motion for a single nonrelativistic particle, using secularity-free perturbation theory. ${ }^{10}$ The constants of motion and bounce frequencies are found, and the time dependence of the particle velocity is determined. Finally, in Sec. III, an estimate of the time dependent damping coefficient is obtained.

\section{PARTICLE ORBITS}

Consider an electromagnetic wave $(\omega, \underset{\mathbf{k}}{\mathbf{k}})$ propagating at an angle $\alpha$ to a magnetic field $\left(\underset{\sim}{\mathbf{B}_{0}}\right)$ in a plasma. If the wave amplitude is not too large, it is reasonable to assume that the propagation characteristics of the wave are primarily determined by the nonresonant particles which form the bulk of the plasma, and that these particles are describable in terms of the linearized theory. Given the additional assumption that $\gamma / \omega<<1$, where $\gamma$ is the damping rate of the wave, the wave fields may be written in the form ${ }^{11}$ 


$$
\begin{aligned}
& \stackrel{\mathbf{B}}{\mathrm{W}}_{\mathrm{W}}(\mathbf{R}, \mathrm{t})={\underset{\sim}{\mathrm{x}}}_{\mathrm{x}} \mathrm{B}_{1} \sin \theta+\left({\underset{\sim}{\mathrm{x}}}_{\mathrm{x}} \times{\underset{\sim}{\mathbf{e}}}_{\mathrm{k}}\right) \mathrm{B}_{2} \cos \theta,
\end{aligned}
$$

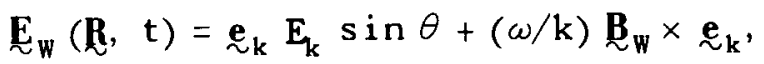

where

$$
\begin{gathered}
\theta=\underset{\sim}{\mathbf{k}} \cdot \underset{\mathbf{R}}{\mathbf{R}}-\omega \mathbf{t}, \\
{\underset{\sim}{\mathbf{k}}}_{\mathbf{k}}=\underset{\mathbf{k}}{\mathbf{k}} / \mathbf{k},
\end{gathered}
$$

and the coordinate system is oriented so that

$$
\begin{aligned}
& {\underset{\sim}{\mathbf{e}}}_{x}=\left(\underset{\sim}{\mathbf{k}} \times \underset{\sim}{\mathbf{B}_{0}}\right) / \mathrm{kB}_{0} \sin \alpha, \\
& \underset{\sim}{\mathbf{e}}=\left(\mathbf{B}_{0} \times \underset{\sim}{\mathbf{e}}\right) / B_{0} \text {, } \\
& {\underset{\sim}{\mathbf{z}}}_{z}=\underset{\sim}{\mathbf{B}}{ }_{0} / \mathrm{B}_{0} \text {. }
\end{aligned}
$$

Two types of wave-particle resonance are possible with obliquely propagating waves. Cyclotron resonance occurs when the rotating wave fields seen by a moving particle turn with a frequency close to a multiple of the particles gyration frequency, $\Omega_{0}$; i.e., when

$$
\omega-k_{z} v_{z}+N \Omega_{0} \approx 0
$$

where $V_{z}$ is the component of the lab frame particle velocity parallel to $B_{0}$, and $\mathrm{N}$ is an integer. In the simplest case $(\mathrm{N}= \pm 1)$, this means that the average value of the angle between the wave electric field and the perpendicular ${ }^{12}$ velocity vector of the gyrating particle changes very slowly. The power delivered to the particle, $\mathbf{E}_{\mathrm{W}} \cdot \underset{\sim}{\mathbf{V}}$, then has a slowly varying component, and large amounts of energy are exchanged. Particles for which $\Varangle \underset{\mathrm{W}}{\mathbf{E}}, \mathbf{V}$ is bounded are said to be trapped. 
Note that it is necessary to speak of the average value of $\underset{\sim}{\underset{\mathbf{E}^{\prime}}{\mathbf{W}^{\prime}}}, \mathbf{V}_{\perp}$ when describing resonance. The plane in which particles gyrate, in zero order, differs from both the plane of polarization of $\mathrm{E}_{\mathrm{w}}$, and the planes of constant phase.

Electrostatic resonance is possible if $\omega / \mathrm{k}_{z}<\mathrm{c}$, where $\omega / \mathrm{k}_{\mathrm{z}}$ is the speed along ${\underset{\sim}{\mathbf{B}_{0}}}_{0}$ of a point of constant phase. In a frame moving with velocity $\left(\omega / \mathrm{k}_{z}\right) \underset{\sim}{\mathbf{e}}$, the wave has only a longitudinal electrostatic component and a transverse magnetic component, and both are stationary. Particles nearly at rest in this frame $\left(\omega-k_{z} V_{z} \sim 0\right)$ experience electrostatic forces which vary only slowly, so that $\underset{\sim}{\mathbf{E}_{\mathrm{W}}} \cdot \underset{\sim}{\mathbf{V}}$ again has a slowly varying component. Such particles may be trapped in the potential wells of the longitudinal field.

\section{Equations of Motion}

If we introduce the velocity variable

$$
\underset{\sim}{\mathbf{v}}=\underset{\sim}{\mathbf{V}}-\left(\omega / \mathbf{k}_{z}\right) \mathbf{e}_{z}
$$

and use (1) and (2), the equation of motion

$$
\mathrm{m} \underset{\sim}{\dot{\mathbf{V}}}=\mathrm{q}\left\{{\underset{\sim}{\mathbf{W}}}_{\mathrm{W}}+\underset{\sim}{\mathbf{V}} \times\left(\stackrel{\boldsymbol{B}}{0}_{0}+\mathbf{B}_{\mathrm{W}}\right)\right\}
$$

can be written in the form

$$
\mathrm{m} \underset{\sim}{\dot{\boldsymbol{v}}}=\mathrm{q}\left\{\mathrm{E}_{\mathrm{k}}^{\prime}{\underset{\sim}{\mathrm{e}}}_{\mathrm{k}} \sin \theta+\underset{\sim}{\mathbf{v}} \times\left({\underset{\sim}{\mathbf{B}_{0}}}+{\underset{\sim}{\mathrm{W}}}_{\mathrm{w}}\right)\right\}
$$

where

$$
\mathrm{E}_{\mathrm{k}}^{\prime}=\mathrm{E}_{\mathrm{k}}+(\omega / \mathrm{k}) \mathrm{B}_{1} \tan \alpha .
$$


The introduction of the variable $\mathbf{v}$ may be viewed as a mathematical change of variables, and does not presuppose the existence of a physical coordinate system moving with velocity $(\omega / \mathrm{k}) \underset{\sim}{\mathbf{e}_{z}}$

It follows easily from (4) that the energy

$$
E=\frac{1}{2} m v^{2}+(q / k) E_{k}^{\prime} \cos \theta
$$

is a constant of the motion.

In a cylindrical coordinate system with axis parallel to ${\underset{\sim}{0}}_{0}$, (4) has the following components :

$$
\begin{aligned}
& \frac{\mathrm{d} \mathrm{v}_{\|}}{\mathrm{dt}}=(\mathrm{q} / \mathrm{m})\left\{\mathrm{E}_{\mathrm{k}}^{\prime} \cos \alpha \sin \theta-\mathrm{v}_{\perp}\left[\mathrm{B}_{-} \cos (\theta-\psi)-\mathrm{B}_{+} \cos (\theta+\psi)\right]\right\} \\
& \frac{\mathrm{d} \mathrm{v}_{\|}}{\mathrm{dt}}=(\mathrm{q} / \mathrm{m})\left\{\left(\frac{1}{2} \mathrm{E}_{\mathrm{k}}^{\prime} \sin \alpha+\mathrm{v}_{\|} \mathrm{B}_{-}\right) \cos (\theta-\psi)-\left(\frac{1}{2} \mathrm{E}_{\mathrm{k}}^{\prime} \sin \alpha+\mathrm{v}_{\|} \mathrm{B}_{+}\right) \cos (\theta+\psi)\right\} \\
& \mathrm{v}_{\perp} \frac{\mathrm{d} \psi}{\mathrm{d} t}=(\mathrm{q} / \mathrm{m})\left\{\mathrm{v}_{\perp}\left[\mathrm{B}_{1 \mathrm{y}} \tan \alpha \cos \theta-\mathrm{B}_{0}\right]+\left(\frac{1}{2} \mathrm{E}_{\mathrm{k}}^{\prime} \sin a+\mathrm{v}_{\|} \mathrm{B}_{-}\right) \sin (\theta-\psi)\right. \\
& \left.+\left(\frac{1}{2} \mathrm{E}_{\mathrm{k}}^{\prime} \sin \alpha+\mathrm{v}_{\|} \mathrm{B}_{+}\right) \sin (\theta+\psi)\right\}
\end{aligned}
$$

where

$$
\begin{aligned}
& v_{\perp}=\left(v_{x}^{2}+v_{y}^{2}\right)^{1 / 2} \\
& \psi=\tan ^{-1}\left(v_{y} / v_{x}\right)
\end{aligned}
$$




$$
\mathrm{B}_{ \pm}=\frac{1}{2}\left(\mathrm{~B}_{1 \mathrm{x}} \pm \mathrm{B}_{1 \mathrm{y}}\right)
$$

and $B_{1 j} \equiv{\underset{W}{W}}_{\sim}{\underset{\sim}{j}}_{j} \cdot$ In addition, we have the equation

$$
\frac{\mathrm{d} \theta}{\mathrm{d} t}=\underset{\sim}{\mathbf{k}} \cdot \mathbf{v}=\mathrm{k}_{\mathrm{y}} \mathrm{v}_{\perp} \text { sin } \psi+\mathrm{k}_{z} \mathrm{v}_{\|}
$$

For nonresonant particles, these equations can be solved to first order in the wave amplitude by integrating the right hand side of equations $(5 a-c)$, along the unperturbed trajectories, defined by

$$
\begin{aligned}
& v_{\|}^{(0)}=v_{\| 0}, \quad v_{\perp}^{(0)}=v_{\perp 0}, \\
& \psi(0)=\psi_{0}-\Omega_{0} t, \\
& \theta(0)=\theta_{0}+\left(k_{y} v_{\perp 0} / \Omega_{0}\right)\left[\cos \left(\psi_{0}-\Omega_{0} t\right)-\cos \psi_{0}\right]+k_{z} v_{\| 0} t,
\end{aligned}
$$

where $\Omega_{0}=\mathrm{qB}_{0} / \mathrm{m}$ and $\mathrm{v}_{\| 0}, \mathrm{v}_{\perp 0}, \psi_{0}, \theta_{0}$ are constants. The first order correction terms calculated in this manner oscillate rapidly in time and remain small.

Orbit expressions calculated in this way for resonant particles contain slowly varying, growing terms which cause the perturbation expansion to break down after a finite time, as the actual particle trajectories drift farther and farther away from the unperturbed trajectories. This breakdown may be avoided by defining a new zero order trajectory. One replaces the constants $\mathrm{v}_{\| 0} \ldots \theta_{0}$ by parameters which vary slowly in time in such a way that the new zero order trajectory remains close to the actual trajectory. ${ }^{10}$ 


$$
\begin{aligned}
& v_{\|}\left(0^{\prime}\right)=\hat{v}_{\|}(\tau), \quad v_{\perp}^{\left(0^{\prime}\right)}=\tilde{v}_{\perp}(\tau), \\
& \psi^{\left(0^{\prime}\right)}=\tilde{\psi}_{0}(\tau)-\Omega_{0} t \text {, } \\
& \theta\left(0^{\prime}\right)=\tilde{\theta}(\tau)+\left[\mathrm{k}_{\mathrm{y}} \widetilde{\mathrm{v}}_{\perp}(\tau) / \Omega_{0}\right] \cdot \cos \left[\tilde{\psi}(\tau)-\Omega_{0} \mathrm{t}\right] \\
& -\left(\mathrm{k}_{\mathrm{y}} \mathrm{v}_{\perp 0} / \Omega_{0}\right) \cos \psi_{0} \\
& +k_{z} \widetilde{v}_{\|}(\tau) t \text {, }
\end{aligned}
$$

where

$$
\tau=\eta_{0}, \eta_{0}<<1 .
$$

For example, if we substitute

$$
\begin{aligned}
& \underset{\sim}{\mathbf{v}}=\mathbf{v}^{\left(0^{\prime}\right)}(\tau, \mathrm{t})+\mathbf{v}^{(1)}(\tau, \mathrm{t})+\cdots \\
& \theta=\theta\left(0^{\prime}\right)(\tau, \mathrm{t})+\theta(1)(\tau, \mathrm{t})+\cdots
\end{aligned}
$$

in (5a), discard terms of second or higher order in the wave amplitude, and Fourier expand the right hand side with respect to $t$, we obtain

$$
\begin{aligned}
\frac{d v_{\| 1}}{d t}= & -\eta_{0} \frac{d \tilde{v}_{\|}(\tau)}{d \tau}+\frac{q}{m} \sum_{n}^{ \pm \infty}\left\{E_{k}^{\prime} \cos a J_{n}\left(\tilde{u}_{\perp}\right)\right. \\
& \left.+\tilde{v}_{\perp}\left[B_{-} J_{n+1}\left(\tilde{u}_{\perp}\right)+B_{+} J_{N-1}\left(\tilde{u}_{\perp}\right)\right]\right\} \sin \left[\left(k_{z} \tilde{v}_{\|}-n \Omega_{0}\right) t+x_{n}\right]
\end{aligned}
$$

where $v_{\| 1} \equiv \underset{\sim}{\mathbf{e}} \cdot{\underset{\sim}{\mathbf{v}}}^{(1)}$

$$
\begin{gathered}
\tilde{\mathrm{u}}_{\perp}(\tau)=\mathrm{k}_{\mathrm{y}} \tilde{\mathrm{v}}_{\perp}(\tau) / \Omega_{0}, \\
x_{\mathrm{n}}(\tau)=\tilde{\theta}(\tau)+\mathrm{n}\left[\tilde{\psi}(\tau)+\frac{\pi}{2}\right]-\left(\mathrm{k}_{\mathrm{y}} \mathrm{v}_{\perp 0} / \Omega_{0}\right) \cos \psi_{0},
\end{gathered}
$$


and $J_{n}$ is the Bessel function of order $n$. When the resonance condition

$$
\mathrm{k}_{\mathrm{z}} \widetilde{\mathrm{v}}_{\|}-\mathrm{N} \Omega_{0} \equiv \mathrm{k}_{\mathrm{z}} \widetilde{\mathrm{V}}_{\|}-\omega+\mathrm{N} \Omega_{0} \sim 0
$$

is satisfied, the acceleration $d v_{\| 1} / d t$ has a slowly varying component (the $\mathrm{n}=$ $\mathrm{N}$ term in the series) which produces secularities in $v_{\| 1}$ unless we choose $\widetilde{\mathrm{v}}_{\|}(\tau)$ such that

$$
\begin{aligned}
\eta_{0} \frac{\mathrm{d} \widetilde{v}_{\| l}(\tau)}{\mathrm{d} \tau} & =\frac{\mathrm{q}}{\mathrm{m}}\left\{\mathrm{E}_{\mathrm{k}}^{\prime} \cos \alpha \mathrm{J}_{\mathrm{N}}\left(\tilde{\mathrm{u}}_{1}\right)\right. \\
& \left.+\tilde{\mathrm{v}}_{\perp}\left[\mathrm{B}_{-} \mathrm{J}_{\mathrm{N}+1}\left(\tilde{\mathrm{u}}_{\perp}\right)+\mathrm{B}_{+} \mathrm{J}_{\mathrm{N}-1}\left(\tilde{\mathrm{u}}_{\perp}\right)\right]\right\} \sin \tilde{\phi}_{\mathrm{N}}(\tau)
\end{aligned}
$$

where

$$
\tilde{\phi}_{N}(\tau)=\left(\mathrm{k}_{\mathrm{z}} \tilde{\mathrm{v}}_{\|}-\mathrm{N} \Omega_{0}\right) \mathrm{t}+\chi_{\mathrm{N}}(\tau)
$$

Given (10), one can integrate (7) with respect to $t$, treating the slow variable $\tau$ as a constant, to obtain

$$
\begin{aligned}
& v_{\| 1} \approx \frac{q}{m} \sum_{n \neq N}^{ \pm \infty}\left\{E_{k}^{\prime} \cos \alpha J_{n}\left(\widetilde{u}_{\perp}\right)+\widetilde{v}_{\perp}\left[B_{-} J_{n+1}\left(\widetilde{u}_{\perp}\right)+B_{+} J_{n-1}\left(\widetilde{u}_{\perp}\right)\right]\right\} \\
& \times \frac{\left\{\cos x_{n}-\cos \left[\left(\mathrm{k}_{\mathrm{z}} \widetilde{\mathrm{v}}_{\|}-\mathrm{n} \Omega_{0}\right) \mathrm{t}+x_{\mathrm{n}}\right]\right\}}{\left(\mathrm{k}_{\mathrm{z}} \widetilde{\mathrm{v}}_{\|}-\mathrm{n} \Omega_{0}\right)}
\end{aligned}
$$

Equations for the remaining slow variables,

$$
\begin{aligned}
\eta_{0} \frac{\mathrm{d} \tilde{v}_{\perp}}{\mathrm{d} \tau}= & -\frac{\mathrm{q}}{\mathrm{m}}\left\{\tilde{v}_{\|}\left[\mathrm{B}_{-} \mathrm{J}_{\mathrm{N}+1}\left(\tilde{u}_{1}\right)+\mathrm{B}_{+} \mathrm{J}_{\mathrm{N}^{-1}}\left(\tilde{\mathrm{u}}_{\perp}\right)\right]\right. \\
& \left.+\frac{1}{2} \mathrm{E}_{\mathrm{k}}^{\prime} \sin \alpha\left[\mathrm{J}_{\mathrm{N}+1}\left(\tilde{u}_{\perp}\right)+\mathrm{J}_{\mathrm{N}^{-1}}\left(\tilde{u}_{\perp}\right)\right]\right\} \sin \tilde{\phi}_{N^{\prime}}
\end{aligned}
$$




$$
\begin{aligned}
& \eta_{0} \frac{\mathrm{d} \tilde{\psi}}{\mathrm{d} \tau}=\frac{\mathrm{q}}{\mathrm{m}}\left\{\frac{\tilde{\mathrm{v}}_{\mathrm{l}}}{\tilde{v}_{\perp}}\left[\mathrm{B}_{-} \mathrm{J}_{\mathrm{N}^{+1}}\left(\tilde{\mathrm{u}}_{\perp}\right)-\mathrm{B}_{+} \mathrm{J}_{\mathrm{N}^{-1}}\left(\tilde{\mathrm{u}}_{\perp}\right)\right]\right. \\
& +\left(\frac{1}{2 \widetilde{v}_{\perp}}\right) E_{k}^{\prime} \sin \alpha\left[J_{N^{-1}}\left(\widetilde{u}_{\perp}\right)-J_{N^{+1}}\left(\widetilde{u}_{\perp}\right)\right] \\
& \left.+B_{1 y} \tan \alpha J_{N}\left(\tilde{u}_{\perp}\right)\right\} \cos \widetilde{\phi}_{N} \\
& \eta_{0} \frac{\mathrm{d} \tilde{\theta}}{\mathrm{d} \tau}=-\eta_{0} \mathrm{t} \mathrm{k}_{z} \frac{\mathrm{d} \tilde{\mathrm{v}}_{\|}}{\mathrm{d} \tau}+\frac{\mathrm{q}}{\mathrm{m}} \mathrm{B}_{1 \mathrm{y}} \tan \alpha\left\{\frac{\mathrm{k}_{\mathrm{z}} \tilde{\mathrm{v}}_{\|}}{\Omega_{0}} \mathrm{~J}_{\mathrm{N}}\left(\tilde{\mathrm{u}}_{\perp}\right)\right. \\
& \left.+\frac{1}{2}\left[J_{N+1}\left(\tilde{u}_{\perp}\right)+J_{N-1}\left(\tilde{u}_{\perp}\right)\right]\right\} \cos \tilde{\phi}_{N}
\end{aligned}
$$

and for the first order corrections to the velocity components $v_{\perp}$ and $\psi$,

$$
\begin{aligned}
& v_{\perp 1}= \frac{q}{m} \sum_{n \neq N}^{ \pm \infty}\left\{\widetilde{v}_{i l}\left[B_{-} J_{n+1}\left(\tilde{u}_{\perp}\right)+B_{+} J_{n-1}\left(\tilde{u}_{\perp}\right)\right]\right. \\
&\left.+\frac{1}{2} E_{k}^{\prime} \sin a\left[J_{n+1}\left(\tilde{u}_{\perp}\right)+J_{n-1}\left(\tilde{u}_{\perp}\right)\right]\right\} \\
& \times\left\{\frac{\cos \left[\left(k_{z} \widetilde{v}_{\|}-n \Omega_{0}\right) t+x_{n}\right]-\cos x_{n}}{\left(k_{z} \widetilde{v}_{\|}-n \Omega_{0}\right)}\right\}, \\
& \psi_{1}= \frac{q}{m} \sum_{n \neq N}^{ \pm \infty}\left\{\frac{\widetilde{v}_{\| 1}\left[B-J_{n+1}\left(\tilde{u}_{\perp}\right)-B_{+} J_{n-1}\left(\tilde{u}_{\perp}\right)\right]}{\widetilde{v}_{\perp}}\right. \\
&+\left(\frac{1}{2 \breve{v}_{\perp}}\right) E_{k}^{\prime} \sin a\left[J_{n+1}\left(\tilde{u}_{\perp}\right) \cdots J_{n-1}\left(\tilde{u}_{\perp}\right)\right]
\end{aligned}
$$




$$
\left.+B_{1 y} \tan \alpha J_{n}\left(\widetilde{u}_{1}\right)\right\}\left\{\frac{\sin \left[\left(k_{z} \widetilde{v}_{\|}-n \Omega_{0}\right) t+x_{n}\right]-\sin x_{n}}{\left(k_{z} \widetilde{v}_{\|}-n \Omega_{0}\right)}\right\},
$$

are obtained in the same way.

\section{Reduction of Phase Space}

Only three different dependent variables $\left(\widetilde{v}_{\|}, \widetilde{v}_{\perp}\right.$, and $\left.\widetilde{\phi}_{N}\right)$ appear in the right hand sides of (10), (13), (14), and (15). With the aid of (9) and (11), equations (14) and (15) may be combined to yield a single equation for $\mathrm{d} \tilde{\phi}_{\mathrm{N}} / \mathrm{d} \tau$. Then, after using the recursion formulas for the Bessel functions and letting $\tau \rightarrow \eta_{0} t$, one obtains the following closed set of equations:

$$
\begin{aligned}
& \frac{d \tilde{v}_{\|}}{d t}=\frac{q}{m}\left\{F_{k}{ }^{\prime} \cos \alpha J_{N}\left(\tilde{u}_{\perp}\right)\right. \\
& \left.+\widetilde{v}_{\perp}\left[B_{1 x}\left(\frac{N \Omega_{0}}{k_{y} \tilde{v}_{\perp}}\right) J_{N}\left(\tilde{u}_{\perp}\right)+B_{1 y} J_{N}^{\prime}\left(\widetilde{u}_{\perp}\right)\right]\right\} \sin \tilde{\phi}_{N} \\
& \frac{\mathrm{d} \widetilde{\mathrm{v}}_{\perp}}{\mathrm{dt}}=-\frac{\mathrm{q}}{\mathrm{m}}\left\{\mathrm{E}_{\mathrm{k}}^{\prime}\left(\frac{\mathrm{N} \Omega_{0}}{\mathrm{k} \widetilde{v}_{\perp}}\right) \mathrm{J}_{\mathrm{N}}\left(\widetilde{\mathrm{u}}_{\perp}\right)\right. \\
& \left.+\widetilde{v}_{i l}\left[B_{1 x}\left(\frac{N \Omega_{0}}{k_{y} \tilde{v}_{\perp}}\right) J_{N}\left(\tilde{u}_{\perp}\right)+B_{1 y} J_{N}^{\prime}\left(\tilde{u}_{\perp}\right)\right]\right\} \sin \tilde{\phi}_{N} \\
& \frac{\mathrm{d} \tilde{\phi}_{\mathrm{N}}}{\mathrm{dt}}=\left(\mathrm{k}_{\mathrm{z}} \widetilde{\mathrm{v}}_{\|}-\mathrm{N} \Omega_{0}\right)+\frac{\mathrm{q}}{\mathrm{m}}\left\{\mathrm{E}_{\mathrm{k}}^{\prime} \sin \alpha\left(\frac{\mathrm{N}}{\widetilde{\mathrm{v}}_{\perp}}\right) \mathrm{J}_{\mathrm{N}}^{\prime}\left(\widetilde{\mathrm{u}}_{\perp}\right)\right. \\
& -B_{1 x} N\left(\frac{\widetilde{v}_{\| 1}}{\widetilde{v}_{\perp}}\right) J_{N}^{\prime}\left(\tilde{u}_{\perp}\right)+B_{1 y}\left[\frac{k_{y} \widetilde{v}_{\| 1}}{\Omega_{0}}\left(1-\frac{N^{2} \Omega_{0}^{2}}{k_{y}^{2} \widetilde{v}_{\perp}^{2}}\right)\right.
\end{aligned}
$$




$$
\left.\left.+N\left(\frac{\Omega_{0}}{k_{z} \tilde{v}_{\perp}}+\frac{k_{y}}{k_{z}}\right)\right] J_{N}\left(\tilde{u}_{1}\right)\right\} \cos \tilde{\phi}_{N}
$$

where $J_{N}^{\prime}\left(\tilde{u}_{\perp}\right) \equiv d J_{N} / d \tilde{u}_{\perp}, \tilde{u}_{\perp}=k_{y} \widetilde{v}_{\perp} / \Omega_{0}$. Once $\widetilde{v}_{\|}, \widetilde{v}_{\perp}$, and $\widetilde{\phi}_{N}$ are known, $\tilde{\theta}$ and $\tilde{\psi}$ may be obtained immediately from (14) and (15) by quadrature.

Exact equations for a particle in resonance with an electrostatic wave propagating along ${\underset{\mathbf{B}}{0}}_{0}$ may be recovered from (18)-(20) by setting $\left(\mathrm{B}_{1 \mathrm{x}}, \mathrm{B}_{1 \mathrm{y}}, \alpha\right.$, $\left.k_{y}, N\right)=0$. Similarly, (18)-(20) reduce to exact equations for a particle in resonance with a parallel propagating, right circularly polarized transverse wave, in the limit $\left(E_{k}^{\prime}, a, k_{y}\right)=0, B_{1 x}=B_{1 y}, N=1$. In these limits there are no rapidly oscillating fields acting on a resonant particle, hence the first order corrections vanish.

\section{Solution of the Slow Variable Equations}

It follows from (18) and (19) that

$$
\frac{d}{d t}\left(\frac{1}{2} m \widetilde{v}^{2}\right)=(q / k) E_{k}^{\prime}\left(k_{z} \widetilde{v}_{\|}-N \Omega_{0}\right) J_{N}\left(\widetilde{u}_{\perp}\right) \sin \tilde{\phi}_{N}
$$

where $\widetilde{v}^{2}=\widetilde{v}_{\|}^{2}+\widetilde{v}_{\perp}^{2}$. But, from $(20)$,

$$
\left(k_{z} \widetilde{v}_{\|}-N \Omega_{0}\right)=\frac{d \widetilde{\phi}_{N}}{d t}+O\left[(\text { amplitude })^{1}\right]
$$

and, from (19),

$$
\frac{\mathrm{d}}{\mathrm{d} t}\left[(\mathrm{q} / \mathrm{k}) \mathrm{E}_{\mathrm{k}}^{\prime} \mathrm{J}_{\mathrm{N}}\left(\tilde{u}_{\perp}\right)\right]=0\left[(\text { amplitude })^{2}\right]
$$


Hence

$$
\begin{aligned}
(\mathrm{q} / \mathrm{k}) \mathrm{E}_{\mathrm{k}}^{\prime}\left(\mathrm{k}_{\mathrm{z}} \tilde{\mathrm{v}}_{\|}-\mathrm{N} \Omega_{0}\right) J_{N}\left(\widetilde{u}_{1}\right) \sin \widetilde{\phi}_{N} \\
=\frac{\mathrm{d}}{\mathrm{dt}}\left[-(\mathrm{q} / \mathrm{k}) \mathrm{E}_{\mathrm{k}}^{\prime} J_{N}\left(\tilde{u}_{\perp}\right) \cos \widetilde{\phi}_{N}\right]+O\left[(\text { amp. })^{2}\right]
\end{aligned}
$$

If we combine (21) and (23) and define

$$
\overline{\mathrm{E}}_{\mathrm{N}} \equiv \frac{1}{2} \mathrm{~m} \widetilde{\mathrm{v}}^{2}+(\mathrm{q} / \mathrm{k}) \mathrm{E}_{\mathrm{k}}^{\prime} \mathrm{J}_{\mathrm{N}}\left(\tilde{\mathrm{u}}_{\perp}\right) \cos \tilde{\phi}_{\mathrm{N}}
$$

we find that $d \overline{\mathrm{E}}_{\mathrm{N}} / \mathrm{dt} \sim \mathrm{O}\left[(\mathrm{amp} .)^{2}\right]$; thus $\overline{\mathrm{E}}_{\mathrm{N}}$ is a constant, to the present order of approximation. $\overline{\mathrm{E}}_{\mathrm{N}}$ is the energy associated with the slow or averaged motion. If one begins by differentiating $\mathrm{m}\left(\tilde{\mathrm{v}}_{\|}-\mathrm{N} \Omega_{0} / \mathrm{k}_{2}\right)^{2} / 2$ instead of $\mathrm{m} \quad \widetilde{v}^{2} / 2$, and then follows the same procedure used above, a second constant is obtained:

$$
\begin{aligned}
\overline{\mathrm{W}}_{\mathrm{N}} & \equiv \frac{1}{2} \mathrm{~m}\left(\widetilde{\mathrm{v}}_{\mathrm{l}}-\mathrm{N} \Omega_{0} / \mathrm{k}_{\mathrm{z}}\right)^{2}+\left(\mathrm{q} / \mathrm{k}_{\mathrm{z}}\right)\left\{\mathrm{E}_{\mathrm{k}}^{\prime} \cos a \mathrm{~J}_{\mathrm{N}}\left(\tilde{\mathrm{u}}_{\perp}\right)\right. \\
& \left.+\widetilde{v}_{\perp}\left[\mathrm{B}_{1 \mathrm{x}}\left(\frac{\mathrm{N} \Omega_{0}}{\mathrm{k}_{\mathrm{y}} \tilde{\mathrm{v}}_{\perp}}\right) \mathrm{J}_{\mathrm{N}}\left(\tilde{\mathrm{u}}_{\perp}\right)+\mathrm{B}_{1 \mathrm{y}} \mathrm{J}_{\mathrm{N}}^{\prime}\left(\tilde{\mathrm{u}}_{\perp}\right)\right]\right\} \cos \tilde{\phi}_{\mathrm{N}}
\end{aligned}
$$

$\overline{\mathrm{E}}_{\mathrm{N}}$ and $\overline{\mathrm{W}}_{\mathrm{N}}$ become exact constants of motion in the limit of parallel propagation, with $\mathrm{N}=0$ for an electrostatic wave and $\mathrm{N}= \pm 1$ for a circularly polarized transverse wave.

With the aid of (25) and (22) the resonance condition may be given somewhat more precisely than before: a particle will be termed resonant if the kinetic 
energy $\mathrm{m}\left(\tilde{\mathrm{v}}_{\|}-\mathrm{N} \Omega_{0} / \mathrm{k}_{\mathrm{z}}\right)^{2} / 2$, which is $\approx \mathrm{m} \mathrm{k}_{\mathrm{z}}^{-2}\left(\mathrm{~d} \tilde{\phi}_{\mathrm{N}} / \mathrm{dt}\right)^{2} / 2$ by (22). is comparable to or less than the amplitude of the effective potential defined by the second term on the right hand side of (25). Thus the resonance condition is

$$
\mathrm{k}_{\mathrm{z}} \widetilde{\mathrm{v}}_{\|}-\mathrm{N} \Omega_{0} \leq \Omega_{0} \mathrm{O}(\eta)
$$

where

$$
\begin{aligned}
\eta\left(\widetilde{v}_{\perp}\right) & =\mid\left(\mathrm{q} \mathrm{k} / \mathrm{m} \Omega_{0}^{2}\right)\left\{\mathrm{E}_{\mathrm{k}}^{\prime} \cos \alpha \mathrm{J}_{\mathrm{N}}\left(\widetilde{\mathrm{u}}_{\perp}\right)\right. \\
& \left.+\widetilde{\mathrm{v}}_{\perp}\left[\mathrm{B}_{1 \mathrm{x}}\left(\frac{\mathrm{N} \Omega_{0}}{\mathrm{k}_{\mathrm{y}} \widetilde{\mathrm{v}}_{\perp}}\right) \mathrm{J}_{\mathrm{N}}\left(\widetilde{\mathrm{u}}_{\perp}\right)+\mathrm{B}_{1 \mathrm{y}} \mathrm{J}_{\mathrm{N}}^{\prime}\left(\widetilde{\mathrm{u}}_{\perp}\right)\right]\right\}\left.\right|^{1 / 2}
\end{aligned}
$$

The appropriateness of this definition can be seen by solving (25) for $\widetilde{\mathrm{v}}_{\|}$and subtracting initial values:

$$
\widetilde{v}_{\|}(\mathrm{t})-\widetilde{v}_{\| 0}=\left.\left[2 \bar{W}_{N} / \mathrm{m}-2 \sigma_{\eta^{2}}\left(\eta \Omega_{0} / \mathrm{k}_{\mathrm{z}}\right)^{2} \cos \widetilde{\phi}_{\mathrm{N}}\right]{ }^{1 / 2}\right|_{0} ^{\mathrm{t}}
$$

where $\sigma_{\eta^{2}}$ is the sign of the coefficient of $\cos \widetilde{\phi}_{N}$ in (25). If (26) is satisfied the quantity in square brackets is of first order, hence

$$
\widetilde{v}_{\|}(t)-\widetilde{v}_{100} \lesssim O\left[(\text { amp. })^{1 / 2}\right]
$$

When (26) is not satisfied $\overline{\mathrm{W}}_{\mathrm{N}}$ is much greater than the term proportional to $\eta^{2}$ in (27), and we can expand the square root, to obtain

$$
\widetilde{v}_{\|}(t)-\widetilde{v}_{\| 0} \lesssim O\left[(\text { amp. })^{1}\right] .
$$

Thus (26) correctly gives the condition for an enhanced or resonant interaction. 
It follows immediately from (24) that

$$
\widetilde{v}^{2}(t)-\widetilde{v}_{0}^{2} \lesssim 0\left[(\text { amp. })^{1}\right]
$$

and it follows from (28) and (29) that

$$
\widetilde{v}_{1}(t)-\widetilde{v}_{\perp 0} \lesssim O\left[(\text { amp. })^{1 / 2}\right]
$$

To lowest order in the square root of the wave amplitude one can ignore the small variation with time of $\vec{v}_{1}(t)$ in terms which are already of first order, in (24), (25), and (18). Thus

$$
\begin{aligned}
& \overline{\mathrm{E}}_{\mathrm{N}} \approx \frac{1}{2} \mathrm{~m} \widetilde{\mathrm{v}}^{2}+(\mathrm{q} / \mathrm{k}) \mathrm{E}_{\mathrm{k}}^{\prime} \mathrm{J}_{\mathrm{N}}\left(\tilde{\mathrm{u}}_{\perp 0}\right) \cos \widetilde{\phi}_{\mathrm{N}} \\
& \overline{\mathrm{W}}_{\mathrm{N}} \approx \frac{1}{2} \mathrm{~m}\left(\widetilde{\mathrm{v}}_{\|}-\mathrm{N} \Omega_{0} / \mathrm{k}_{\mathrm{z}}\right)^{2}+\mathrm{m} \sigma_{\eta^{2}}\left(\eta_{0} \Omega_{0} / \mathrm{k}_{\mathrm{z}}\right)^{2} \cos \widetilde{\phi}_{\mathrm{N}} \\
& \frac{\mathrm{d} \widetilde{\mathrm{v}}_{\|}}{\mathrm{d} \mathrm{t}} \approx \frac{\Omega_{0}^{2}}{\mathrm{k}_{\mathrm{z}}} \sigma_{\eta^{2}} \eta_{0}^{2} \sin \widetilde{\phi}_{\mathrm{N}}
\end{aligned}
$$

where $\eta_{0} \equiv \eta\left(\tilde{\mathrm{v}}_{\perp 0}\right)$.

The path of a particle in the two dimensional phase space with coordinates $\widetilde{\phi}_{N}, \widetilde{v}_{\|}$can be determined by solving (32) for $\widetilde{\phi}_{N}$ and plotting $\widetilde{\phi}_{N}$ versus $\widetilde{v}_{\|}$. A typical set of such paths, for a fixed value of $\widetilde{v}_{10}$ and several different values of $\vec{W}_{N}$, is illustrated in Fig. 1. Note that the angle $\widetilde{\phi}_{N}$ is bounded for particles following the closed orbits. Such particles are said to be trapped. 
Eq. (32) may also be used to eliminate $\widetilde{\phi}_{N}$ from (33). The resulting equation may be written in the form

$$
\frac{\mathrm{d} x}{\mathrm{~d} s}= \pm\left[\left(1-x^{2}\right)\left(x^{2}+\mathrm{r}^{2}-1\right)\right]^{1 / 2}
$$

where

$$
\begin{aligned}
x & =\left(\mathrm{r} / 2 \eta_{0}\right)\left(\mathrm{k}_{\mathrm{z}} \widetilde{\mathrm{v}}_{\|} / \Omega_{0}-\mathrm{N}\right) \\
\mathrm{d} \mathrm{s} & =-\left(\eta_{0} \Omega_{0} / \mathrm{r}\right) \mathrm{d} \mathrm{t} \\
\mathrm{r}^{2} & =2\left(1+\mathrm{k}_{\mathrm{z}}^{2} \overline{\mathrm{w}}_{\mathrm{N}} / \mathrm{m} \eta_{0}^{2} \Omega_{0}^{2}\right)^{-1}
\end{aligned}
$$

Eq. (34) is the equation satisfied by the Jacobian elliptic function $\mp \operatorname{dn}(s, r)$. Thus

$$
\widetilde{v}_{\|}(t)=N \Omega_{0} / k_{z} \mp\left(2 \eta_{0} \Omega_{0} / r k_{z}\right) \operatorname{dn}\left[\left(\eta_{0} \Omega_{0} / r\right)\left(t_{+}-t\right), r\right]
$$

The function $\mathrm{dn}(\mathrm{s}, \mathrm{r})$ is periodic in $\mathrm{s}$. The period of the motion, for untrapped particles, is

$$
\mathrm{T}_{\mathrm{N}}=2 \mathrm{rK}(\mathrm{r}) / \eta_{0}\left(\widetilde{\mathrm{v}}_{\perp 0}\right) \Omega_{0}
$$

where $\mathrm{K}(\mathrm{r})$ is the complete elliptic integral of the first kind. For untrapped particles with velocities greater (less) than $\mathrm{N} \Omega_{0} / \mathrm{k}_{z}$ one chooses the lower (upper) sign in (36). The phase constant $t_{+}$is any time at which the quantity $\left|\widetilde{v}_{\|}-\mathrm{N} \Omega_{0} / \mathrm{kz}\right| \cdot$ has its maximum value. The parameter $\mathrm{r}$ classifies the particle orbits according to type. $r$ is greater than one for trapped particles, with $r \rightarrow \infty$ for particles in the center of the nested set of closed orbits in Fig. 1. r has the value unity for particles on the separatix and is less than one for untrapped particles, with $\mathrm{r} \lesssim \mathrm{O}\left[(\mathrm{amp})^{1 / 2}\right]$ for nonresonant particles. ${ }^{13}$ 
Strictly speaking, (36) is valid for both trapped and untrapped particles, since $\operatorname{dn}(s, r)$ is defined by analytic continuation when $r>1$. It is convenient, however, to reserve the use of (36) in its present form for untrapped particles $(r<1)$, and to use the identity dn $(s, r) \equiv \mathrm{cn}(\mathrm{rs}, 1 / \mathrm{r})$ when $\mathrm{r} \geq 1$. Thus

$$
\begin{gathered}
\widetilde{\mathrm{v}}_{\|}(\mathrm{t})=\mathrm{N} \Omega_{0} / \mathrm{k}_{\mathrm{z}}+\left(2 \eta_{0} \Omega_{0} / \mathrm{rk}_{\mathrm{z}}\right) \mathrm{cn}\left[\eta_{0} \Omega_{0}\left(\mathrm{t}_{+}-\mathrm{t}\right), 1 / \mathrm{r}\right] \\
\mathrm{T}_{\mathrm{N}}=4 \mathrm{~K}(1 / \mathrm{r}) / \eta_{0}\left(\widetilde{\mathrm{v}}_{10}\right) \Omega_{0}
\end{gathered}
$$

for trapped particles. We have omitted the upper sign on the last term in (36), in deriving (38). Adding one half period to $t_{+}$changes the sign of the elliptic function cn, hence no generality is lost through this omission.

After solving (32) for $\widetilde{\phi}_{\mathrm{N}}$ and using (35) and (36), one has

$$
\tilde{\phi}_{N}(t)=\cos ^{-1}\left(\left(2 \sigma_{\eta^{2}} / \mathrm{r}^{2}\right)\left\{1-\mathrm{d} \mathrm{n}^{2}\left[\left(\eta_{0} \Omega_{0} / \mathrm{r}\right)\left(\mathrm{t}-\mathrm{t}_{+}\right), \mathrm{r}\right]\right\}-\sigma_{\eta^{2}}\right)
$$

The time dependence of $\widetilde{v}_{\perp}$ is found by solving (31) for $\widetilde{v}_{\perp}^{2}$, subtracting initial values, and using (36) and (40). After taking and expanding the square root of the resulting equation, one has

$$
\widetilde{v}_{\perp}(t) \approx \widetilde{v}_{\perp 0} \pm\left.\left(2 \mathrm{~N} \eta_{0} \Omega_{0}^{2} / \mathrm{rk}_{z}^{2} \widetilde{v}_{\perp 0}\right) \mathrm{dn}\left[\left(\eta_{0} \Omega_{0} / \mathrm{r}\right)\left(\mathrm{t}-\mathrm{t}_{+}\right), \mathrm{r}\right]\right|_{0} ^{\mathrm{t}}
$$

to lowest order in (amp.) $)^{1 / 2}$. Again, one can use the identity $\mathrm{dn}(\mathrm{s}, \mathrm{r}) \equiv \mathrm{cn}(\mathrm{rs}, 1 / \mathrm{r})$ for trapped particles. 
In summary, the major effect of the resonance on the motion of single particles is to induce the particles to execute large amplitude ( $\left.\sim \operatorname{amp} .{ }^{1 / 2}\right)$, long period ( amp. $^{1 / 2}$ ) oscillations about their zero order trajectories. Superimposed on these large motions are rapid, small amplitude oscillations.

\section{SATURATION OF RESONANCE DAMPING}

The physical mechanism underlying the Landau damping of electrostatic waves was described by Dawson in $1961 .^{14}$ Dawson demonstrated that the damping was due to the presence of resonant particles which move with velocities very close to the wave velocity. These particles exchange energy with the wave on a time scale long compared with the period $2 \pi / \omega$. If the resonant particles are initially distributed or bunched in phase space in such a way that more particles gain than lose energy, then the wave damps, by conservation of energy. The kinetic energy of a resonant particle in a sinusoidal potential oscillates in time, but the period of oscillation approaches infinity if the wave amplitude is infinitesimal. Thus a very small amplitude wave damps monotonically.

If the wave amplitude is large the periodic nature of the particle motion can not be neglected. This situation has been treated by $O^{\prime}$ Neil. ${ }^{1}$ After a time of the order of a half period of a typical resonant particle's motion, and if the wave amplitude has not changed too much in the meantime, most of the particles which were initially gaining energy begin to lose energy, and vice versa. The damping coefficient then changes sign, and the wave amplitude begins to oscillate. 
The bunching of resonant particles in phase space, required for a net exchange of energy between wave and plasma, cannot be maintained indefinitely. The frequency of oscillation of a particle in a potential well of the wave depends on the particle's energy, and the spectrum of oscillation frequencies is broad. Thus, phase mixing of resonant particles eventually causes the damping coefficient to vanish entirely. In the absence of instabilities one is left with a wave of constant amplitude.

The cyclotron damping of a finite amplitude whistler wave propagating parallel to a background magnetic field has been shown to saturate in the same way. ${ }^{2}$

In the preceding section we have shown that the motion of particles in resonance with an obliquely propagating wave exhibits, to lowest order in (amp. $)^{1 / 2}$, all of the features relevant to the nonlinear damping process described above. That is:

1. resonant particles exchange energy with the wave on a time scale long compared with $2 \pi / \omega$;

2. the kinetic energy of a resonant particle in an oblique wave is periodic in time;

3. the spectrum of resonant particle oscillation frequencies is broad. One therefore expects that the damping of a finite amplitude oblique wave should also vanish after some time, to lowest order. 
In this section we shall describe the resonance damping of an obliquely propagating wave in the nonlinear regime. Our purpose is not to provide a fully detailed picture of the damping process, but rather to determine the time dependence of the nonlinear damping coefficient in a simple, somewhat heuristic way. Specifically, we shall assume that the damping coefficient obtainable from the linearized theory,

$$
\gamma_{L}=\sum_{N} \gamma_{L_{N}}
$$

is known, and describe the nonlinear coefficient $\gamma(t)$ in terms of functions $h_{N}(t)$, defined by the relation

$$
\int_{0}^{t} \gamma(t) d t=\sum_{N} \gamma_{L N} h_{N}(t) .
$$

The index $\mathrm{N}$ numbers the contributions from the various resonances.

Following the method used in Ref's. 1 and 2, we begin with the energy conservation equation. If the amplitude parameters $E_{k}, B_{i}$ have a time dependence of the form

$$
A(t)=A_{0} \exp \left[\int_{0}^{t} \gamma(t) d t\right],
$$

then the energy equation in the lab frame is

$$
2 Q_{1} \gamma(t)+P_{n r}(t)+\sum_{N}\left[P_{r}(t)\right]_{N}=0
$$


where

$$
\mathrm{Q}_{1}=2 \pi \epsilon_{0}\left[\mathrm{E}_{\mathrm{k}}^{2}+\left(\frac{\omega^{2}}{\mathrm{k}^{2}}+\mathrm{c}^{2}\right)\left(\mathrm{B}_{1}^{2}+\mathrm{B}_{2}^{2}\right)\right]
$$

The quantity $2 Q_{1} \gamma$ is the time rate of change of the wave energy per wavelength. $P_{n r}$ and $\Sigma\left(P_{r}\right)_{N}$ represent the rate of energy absorption per wavelength by nonresonant and resonant particles, respectively.

The time scale on which the wave amplitude varies is assumed to be much longer than the period of oscillation of a nonresonant particle, hence adiabatic invariants exist which relate the constants of motion of a nonresonant particle at any given time to the instantaneous value of the wave amplitude. If follows from this that the total kinetic energy per wavelength of nonresonant particles depends on time only through the wave amplitude. Hence

$$
P_{n r}(t) \equiv \frac{d}{d t}(\text { K. E. })_{n r}=Q_{2} \gamma(t)
$$

where $Q_{2}$ is a function of the wave amplitude and various constants.

After using (44) in (43), the latter equation may be solved for $\gamma(t)$.

$$
\gamma(\mathrm{t})=-\sum_{\mathrm{N}}\left[\left(\mathrm{P}_{\mathrm{r}}\right)_{\mathrm{LN}} /\left(\mathrm{Q}_{1}+\mathrm{Q}_{2}\right)\right]\left\{\left[\mathrm{P}_{\mathrm{r}}(\mathrm{t})\right]_{\mathrm{N}} /\left(\mathrm{P}_{\mathrm{r}}\right)_{\mathrm{LN}}\right\}
$$

where $\left(P_{r}\right)_{L N}$ is the value of $\left[P_{r}(t)\right]_{N}$ obtainable from the linearized theory. If we recall that linearized theory provides an adequate description of the behavior of nonresonant particles, in the present approximation, it follows from a comparison of (41), (42), and (45) that 


$$
\begin{aligned}
& -\left(P_{r}\right)_{L N} /\left(Q_{1}+Q_{2}\right)=\gamma_{L N} \\
& {\left[P_{r}(t)\right]_{N} /\left(P_{r}\right)_{L N}=\frac{d}{d t} h_{N}(t) .}
\end{aligned}
$$

The rate of energy absorption by resonant particles is given by the relation

$$
\left[P_{r}(t)\right]_{N}=\frac{d}{d t}\left[\frac{1}{2} m \iiint_{V_{N}} d^{3} v \int_{0}^{2 \pi} d \theta\left(v^{2}+\frac{2 \omega v_{\|}}{k_{z}}+\frac{\omega^{2}}{k_{z}^{2}}\right) f(\theta, \underset{v}{v}, t)\right]
$$

The quantity in parenthesis in the integrand above is the square of the lab frame velocity. $f$ is the distribution function, and $V_{N}$ is the volume in velocity space associated with the Nth resonance. To estimate the relative magnitude of the various terms in (47), it is helpful to write $\left[P_{r}(t)\right]_{N}$ in the alternative form

$$
\begin{aligned}
{\left[P_{r}(t)\right]_{N} } & =\frac{1}{2} m \iiint_{v_{N}} d^{3} v_{0} \int_{0}^{2 \pi} d \theta_{0} \frac{\partial}{\partial t}\left[v^{2}\left(\theta_{0}, \mathbf{v}_{0}, t\right)\right. \\
& \left.+2 \omega v_{\|}\left(\theta_{0}, v_{0}, t\right) / k_{z}+\omega^{2} / k_{z}^{2}\right] f\left(\theta_{0}, \mathbf{v}_{0}, 0\right)
\end{aligned}
$$

The constant $\omega^{2} / \mathrm{k}_{\mathrm{z}}^{2}$ vanishes under the time derivative and makes no contribution. If saturation occurs before the wave amplitude has changed very much the approximate trajectories developed in Sec. II may be used to express $v^{2}\left(\theta_{0}, \underset{\sim}{\mathbf{v}_{0}}\right.$, t) and $v_{\|}\left(\theta_{0}, \underset{\sim}{v_{0}}, t\right) . v^{2}$ and $v_{\|}$may be separated into fast and slow parts. As far as damping is concerned, the important effect of the resonance is to produce the large, slow deviations from the zero order particle trajectories described 
by the slow variables. Thus we consider only the slow parts. It follows from (18) that $d \widetilde{v}_{\|} / d t$ is a first order quantity, while (21) and (36) indicate that $d \tilde{v}^{2} / d t$ is of the order (amp.) $)^{3 / 2}$. (After integration the $\widetilde{v}_{\mathfrak{i} \mid}$ term is of order (amp.) $)^{2}$, while the $\widetilde{v}^{2}$ term is of order (amp. $)^{5 / 2}$.) Thus, to lowest order,

$$
\left[P_{r}(t)\right]_{N} \approx \frac{d}{d t}\left[\frac{m \omega}{2 k_{z}} \iiint_{v_{N}} d^{3} \tilde{v} \int_{0}^{2 \pi} d \tilde{\theta} \tilde{v}_{\|} f(\tilde{\theta}, \tilde{v}, t)\right]
$$

We shall assume that $f$ is Maxwellian when the wave is "turned on" at $t=0$. The small deviation from thermal equilibrium required to support the wave initially will be neglected. In general, this initial perturbation phase mixes away rapidly and makes no significant contribution to the damping. In a collisionless plasma the phase space density in the vicinity of a given particle is conserved, hence

$$
\begin{aligned}
\mathrm{f}(\tilde{\theta}, \tilde{\mathbf{v}}, \mathrm{t}) & =\mathrm{A} \exp \left\{-\left[\tilde{v}_{0}^{2}(\tilde{\theta}, \underset{\sim}{\tilde{\mathbf{v}}}, \mathrm{t})+2 \omega \tilde{v}_{\| 0}(\tilde{\theta}, \underset{\sim}{\tilde{\mathbf{v}}}, \mathrm{t}) / \mathrm{k}_{\mathrm{z}}\right.\right. \\
& \left.\left.+\omega^{2} / \mathrm{k}_{\mathbf{z}}^{2}\right] / \mathrm{V}_{\mathrm{T}}^{2}\right\}
\end{aligned}
$$

where $\widetilde{v}_{0}^{2}, \widetilde{v}_{\| 0}$ are the values of $\widetilde{v}^{2}, \widetilde{v}_{\|}$at $t=0$ for a particle at $\tilde{\theta}, \widetilde{v}$ at time $t$, and $\mathrm{A}$ is a normalization constant. The right hand side of (49) will be expanded in the wave amplitude. It follows from (28) and (29) that the lowest order time dependent term in the expansion will be of order (amp. $)^{1 / 2}$ and will come from $v_{\| 0}(\tilde{\theta}, \underset{\sim}{\tilde{v}}, t)$. To this order, $\widetilde{v}_{0}^{2} \approx \widetilde{v}^{2}$. Thus, after setting $\widetilde{v}_{\| 0}$ equal to $N \Omega_{0} / k_{z}$ plus the small quantity $\left(\widetilde{\mathrm{v}}_{\| 0}-\mathrm{N} \Omega_{0} / \mathrm{k}_{\mathrm{z}}\right)$ and expanding, we have 


$$
\begin{aligned}
\mathrm{f}(\tilde{\theta}, \tilde{\sim}, \mathrm{t}) & =\mathrm{A} \exp \left\{-\left[\mathrm{v}^{2}+\left(\omega^{2}+2 \mathrm{~N} \omega \Omega_{0}\right) / \mathrm{k}_{\mathrm{z}}^{2}\right] / \mathrm{V}_{\mathrm{T}}^{2}\right\} \\
& \times\left[1-\left(2 \omega / \mathrm{k}_{\mathrm{z}} \mathrm{V}_{\mathrm{T}}^{2}\right)\left(\tilde{\mathrm{v}}_{\| 0}-\mathrm{N} \Omega_{0} / \mathrm{k}_{\mathrm{z}}\right)-\cdots\right]
\end{aligned}
$$

The integral in (48) can be evaluated if one additional simplifying assumption is made. We shall assume that the perpendicular velocity of a typical resonant particle is small compared with $\Omega_{0} / \mathrm{k}_{\mathrm{y}}$, and neglect terms of order $\left(\mathrm{k}_{\mathrm{y}} \widetilde{\mathrm{v}}_{\perp} / \Omega_{0}\right)^{2} \equiv$ $\widetilde{\mathrm{u}}_{\perp}^{2} \cdot$ Thus, for $\mathrm{N}=0,(26 \mathrm{~b})$ gives

$$
\eta_{0} \equiv \eta\left(\tilde{\mathrm{\nu}}_{L_{0}}\right)=\left|\mathrm{qk}_{\mathrm{z}} \mathrm{E}_{\mathrm{k}}^{\prime} \cos \alpha / \mathrm{m} \Omega_{0}^{2}\right|^{1 / 2}+0\left(\tilde{\mathrm{u}}_{\perp 0}^{2}\right)
$$

When $N= \pm 1, \eta_{0}$ is proportional to $\tilde{\mathrm{v}}_{\perp 0}^{1 / 2}$. For a resonant particle $\widetilde{\mathrm{v}}_{10}=\left[\widetilde{\mathrm{v}}^{2}-\right.$ $\left.\left(\mathrm{N} \Omega_{\mathrm{o}} / \mathrm{kz}\right)^{2}\right]^{1 / 2}+\mathrm{O}\left[(\mathrm{amp} .)^{1 / 2}\right]$. Thus, when $\mathrm{N}= \pm 1$

$$
\begin{aligned}
\eta_{0} & =\left|\left(q / m \Omega_{0}\right)\left[\frac{1}{2}\left(B_{1 y} \pm B_{1 x}\right)+k_{y} E_{k}^{\prime} \cos \alpha / 2 \Omega_{0}\right]\right|^{1 / 2} \\
& \times\left|\left(k_{z} \widetilde{v} / \Omega_{0}\right)^{2}-1\right|^{1 / 4}+O\left[(a m p \cdot)^{3 / 2}, \tilde{u}_{10}^{2}\right] .
\end{aligned}
$$

When $|\mathrm{N}| \geq 2, \eta_{0} \leq 0\left(\tilde{\mathrm{u}}_{10}^{2}\right)$. It follows from this last relationship and (36), (45), (48), and (50) that

$$
\gamma(\mathrm{t})=\sum_{N=-1}^{+1} \gamma_{L N} \frac{\mathrm{dh}_{\mathrm{N}}}{\mathrm{dt}}+\mathrm{O}\left(\left\langle\tilde{\mathrm{u}}_{L 0}^{2}\right\rangle\right)
$$

When $N= \pm 1$, the oscillatory part of the motion described by (35), (36), (38), and (52) is identical to the approximate parallel oscillatory motion of a particle 
in resonance with a whistler wave propagating along the magnetic field, if the wave number and amplitude of the whistler are given by

$$
\begin{aligned}
\mathrm{k}_{\text {eff. }} & =\mathrm{k}_{\mathrm{z}} \\
\mathrm{B}_{1, \text { eff. }} & =\frac{1}{2}\left(\mathrm{~B}_{1 \mathrm{y}} \pm \mathrm{B}_{1 \mathrm{x}}\right)+\mathrm{k}_{\mathrm{y}} \mathrm{E}_{\mathrm{k}}^{\prime} \cos \alpha / 2 \Omega_{0} .
\end{aligned}
$$

[See Eq.'s. (21a), (21b), and (22) of Ref. 2.] In addition, (48) and (50) are identical in form to the corresponding equations $\left[(25),\left(26^{\prime}\right)\right.$ and the first eq. in Appendix B of Ref. 2.] for the parallel propagating whistler. The rate of energy absorption by particles in resonance with a parallel propagating whistler is calculated explicitly in Ref. 2. We can therefore obtain expressions for $\left[\mathrm{P}_{\mathrm{r}}(\mathrm{t})\right]_{ \pm 1}$ by making appropriate generalizations of the constants in the results of this earlier work.

Similarly, it is easily shown that (35), (36), (38), and (51) make the evaluation of (48) in the case $\mathrm{N}=0$ mathematically equivalent to the calculation of $P_{r}(t)$ for a finite amplitude electrostatic wave. We can obtain $\left[\mathrm{P}_{\mathrm{r}}(\mathrm{t})\right]_{0}$ by making appropriate generalizations of the constants in $\mathrm{O}^{\prime} \mathrm{Neil}^{1}{ }^{1}$ result.

Finally, it is demonstrated in Ref's. 1 and 2 that, for $\mathrm{N}=0, \pm 1$,

$$
\left(\mathrm{P}_{\mathrm{r}}\right)_{\mathrm{LN}}=\underset{t / \tau_{\mathbf{N}} \rightarrow 0}{1 \text { imit }}\left[\mathrm{P}_{\mathbf{r}}(\mathrm{t})\right]_{N}
$$

where $\tau_{N}$ is the period of oscillation (slow) of a typical resonant particle. 
After following the procedure for finding $\left[P_{r}(t)\right]_{0, \pm 1}$ outlined above and using (46) and (54), we have

$$
\begin{aligned}
\mathrm{h}_{0}(\mathrm{t})=\tau_{0} \sum_{\mathrm{n}=0}^{\infty} \frac{64}{\pi} \int_{0}^{1} \mathrm{dr}\left\{\frac{2 \pi\left(1-\cos \left[\frac{\pi n \mathrm{t}}{\mathrm{rK}(\mathrm{r}) \tau_{0}}\right]\right)}{\mathrm{r}^{4} \mathrm{~K}(\mathrm{r})\left(1+\mathrm{q}^{2 \mathrm{n}}\right)\left(1+\mathrm{q}^{-2 \mathrm{n}}\right)}\right. \\
\left.+\frac{2 \pi \mathrm{r}\left(1-\cos \left[\frac{(2 \mathrm{n}+1) \pi \mathrm{t}}{\left.\left.2 \mathrm{~K}(\mathrm{r}) \tau_{0}\right]\right)}\right.\right.}{\mathrm{K}(\mathrm{r})\left(1+\mathrm{q}^{2 \mathrm{n}+1}\right)\left(1+\mathrm{q}^{-2 n-1}\right)}\right\}
\end{aligned}
$$

where $q=\exp \left\{-\pi K\left(\left[1-r^{2}\right]^{1 / 2}\right) / K(r)\right\}$ and

$$
\tau_{0}=\left|(q k / m) E_{k}^{\prime} \cos ^{2} a\right|^{-1 / 2}
$$

and

$$
\begin{aligned}
\mathrm{h}_{ \pm 1}(\mathrm{t}) & =2^{9} \pi^{1 / 2} \frac{\mathrm{C}_{0} \zeta_{0}}{\mathrm{C}_{1}} \tau_{ \pm 1} \\
& \times \int_{0}^{1} \mathrm{dr}\left(\sum_{\mathrm{n}=1}^{\infty} \frac{1-\exp \left\{-\left[\left(\mathrm{n} \pi / 2 \mathrm{C}_{1} \zeta_{0} \mathrm{rK}\right)\left(\mathrm{t} / \tau_{ \pm 1}\right)\right]^{2}\right\} \cos \left[(\mathrm{n} \pi / \mathrm{rK})\left(\mathrm{t} / \tau_{ \pm 1}\right)\right]}{\mathrm{r}^{4} \mathrm{~K}(\mathrm{r})\left(1+\mathrm{q}^{2 \mathrm{n}}\right)\left(1+\mathrm{q}^{-2 \mathrm{n}}\right)}\right. \\
& \left.+\sum_{n=0}^{\infty} \frac{\left[1-\exp \left(-\left\{\left[(2 \mathrm{n}+1) \pi / 4 \mathrm{C}_{1} \zeta_{0} \mathrm{~K}\right]\left(\mathrm{t} / \tau_{ \pm 1}\right)\right\}^{2}\right) \cos \left\{[(2 \mathrm{n}+1) \pi / 2 \mathrm{~K}]\left(\mathrm{t} / \tau_{ \pm 1}\right)\right\}\right] \mathrm{r}}{\mathrm{K}(\mathrm{r})\left(1+\mathrm{q}^{2 \mathrm{n}+1}\right)\left(1+\mathrm{q}^{-2 \mathrm{n}-1}\right)}\right)
\end{aligned}
$$


where

$$
\tau_{ \pm 1}=\left|\left(\mathrm{qk}_{\mathrm{z}} \mathrm{v}_{\mathrm{T}} \zeta_{0}^{2} / \mathrm{m}\right)\left[\frac{1}{2}\left(\mathrm{~B}_{1 \mathrm{y}} \pm \mathrm{B}_{1 \mathrm{x}}\right)+\mathrm{k}_{\mathrm{y}} \mathrm{E}_{\mathrm{k}}^{\prime} \cos \alpha / 2 \Omega_{0}\right]\right|^{-1 / 2}
$$

and $\zeta_{0} \approx 1.1, \mathrm{C}_{0} \approx .41, \mathrm{C}_{1} \approx 3.1$

When these results are substituted into (53) our estimate of the damping coefficient is complete. The time variation of $h_{0}(t)$ has been described in the introduction to this section. The behavior of the functions $h_{1}(t)$ and $h_{-1}(t)$ is similar, and is sketched in Fig.'s 3 and 4 of Ref. 2 .

The asymptotic values $h_{0, \pm 1}(\infty)$ have been calculated in the papers quoted above. One finds that

$$
\int_{0}^{\infty} \gamma(t) d t \approx 2 \sum_{N=-1}^{+1} \gamma_{L N} \tau_{N}
$$

In deriving (55) and (56) we have assumed that saturation occurs before the wave amplitude changes appreciably. This assumption is justified if $\int_{0}^{\infty} \gamma \mathrm{dt} \ll 1$.

In a multi-component plasma, these results represent the contribution of any one species to the total damping coefficient.

\section{SUMMARY}

We have found approximate solutions, of relatively simple form, to the equations of motion for a particle in resonance with a small finite amplitude wave 
propagating at an angle to a magnetic field in a plasma. These solutions can be applied to the study of several important effects which involve resonant particles.

One such effect, resonance damping, has been considered here. We have shown that the resonance damping coefficient for a finite oblique wave vanishes after a few bounce times, leaving, in the absence of instabilities, an undamped wave.

\section{ACKNOW LEDGMENT}

This research was performed while the author held a National Research Council Resident Research Associateship. 


\section{REFERENCES}

1. T. O'Neil, Phys. Fluids, 8, 2255 (1965).

2. P. Palmadesso and G. Schmidt, Phys. Fluids, 14, 1411 (1971).

3. W. L. Kruer, J. M. Dawson, and R. N. Sudan, Phys. Rev. Letters, 23, 838 (1969).

4. M. V. Goldman, Phys. Fluids, 13, 1281 (1970).

5. W. M. Manheimer, Phys. Rev. A, 3, 1402 (1971).

6. P. Palmadesso and G. Schmidt, Phys. Fluids, 15, 485 (1972).

7. R. N. Sudan and E. Ott, J. Geophys. Res., 76, 4463 (1971).

8. K. B. Dysthe, J. Geophys. Res., 76, 6915 (1971).

9. C. S. Roberts and S. J. Buchsbaum, Phys. Rev., 135A, 381 (1964).

10. N. Bogoliubov and Y. A. Mitropolskii, Asymptotic Methods in the Theory of Nonlinear Oscillations, Gordon and Breach Science Publishers, New York, 1961 [translated from the Russian].

11. V. L. Ginzburg, The Propagation of Electromagnetic Waves in Plasmas, Addison-Wesley Publishing Co., Reading, 1964 [translated from the Russian] :

12. We are using the terms "perpendicular" and "parallel" to describe orientations with respect to ${\underset{\sim}{\mathbf{B}}}_{0}$, and "transverse" and "longitudinal" to describe orientations with respect to $\underset{\sim}{\mathbf{k}}$.

13. To verify that $\mathbf{r}=1$ on the separatrix set $\tilde{\phi}_{N}=\pi\left(1-\sigma_{\eta^{2}}\right) / 2$ and $\widetilde{v}_{\|}=N \Omega_{0} / \mathrm{kz}$ in (32) and use the result in (35). (See Fig. 1.) $\bar{W}_{N}$ increases as one moves away from the trapping region and decreases as one moves into it. 
14. J. M. Dawson, Phys. Fluids, 4, 869 (1961).

15. The notation of Ref. 2 is similar, but not identical, to that used here. 


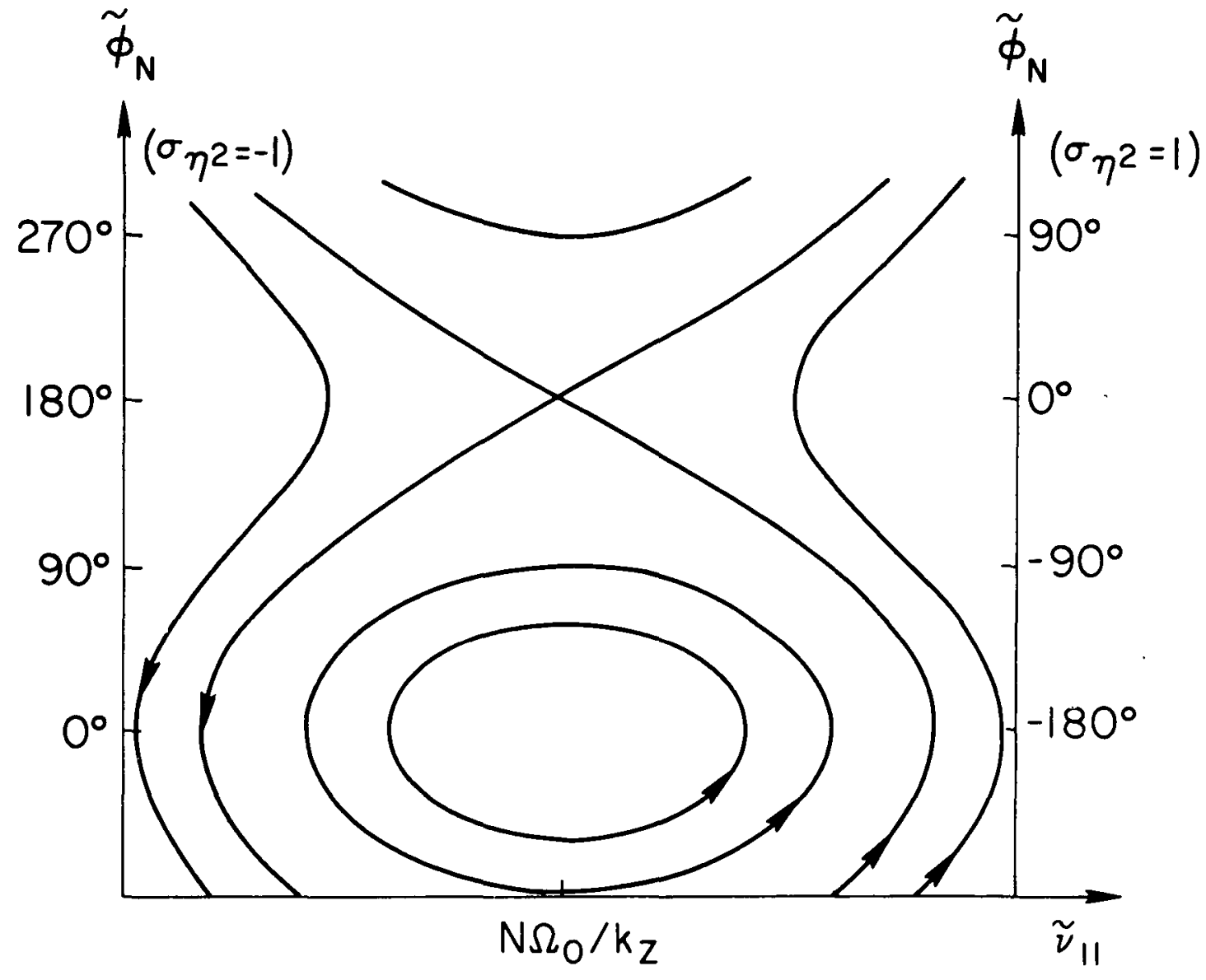

Figure 1. Phase plane trajectories for typical trapped and untrapped resonant particles. 